\title{
Sobre el estudio empírico de la solidaridad: aproximaciones conceptuales y metodológicas*
}

\author{
Ignacio Madero Cabib \\ Pontificia Universidad Católica de Chile, Santiago, Chile. Email: imadero@uc.cl \\ Juan Carlos Castillo \\ Pontificia Universidad Católica de Chile, Santiago, Chile. Email: jcastillov@puc.cl
}

\begin{abstract}
Resumen: La solidaridad, entendida como una operación social y comunicativa destinada a mantener la cohesión de las personas en el marco de la sociedad moderna, pone en evidencia la permanencia de un orden social estratificado que produce exclusión de ciertos derechos sociales. El presente artículo, intenta discutir más a fondo esta definición ofreciendo un análisis empírico y multidimensional de las conductas solidarias en Chile. Mediante análisis bivariados y de MANOVA de los datos de la "Encuesta de solidaridad 2010" (N=1389) generada por el centro de medición MIDE UC de la Pontificia Universidad Católica, llegamos a la conclusión que existen ciertas variables del esquema de estratificación social que en la actualidad predicen y direccionan la orientación de la solidaridad en Chile. Esto sugiere que la solidaridad no solamente responde a una esquematización estratificada de la sociedad moderna, sino además agrega que diferentes ejes de estratificación social influencian las dimensiones de la solidaridad
\end{abstract}

Palabras Clave: Solidaridad, Modernidad, Estratificación Social, Exclusión Social.

\section{About the empirical study of solidarity: conceptual and methodological approaches}

\begin{abstract}
Solidarity, understood as a social and communicative operation destined to maintain people's cohesion in the context of modern society, evidences the permanence of a stratified social order that produces exclusion from certain social rights. This article attempts to further discuss this definition by offering an empirical and multidimensional analysis of solidarity conducts in Chile. Through an univariate analysis and MANOVA analysis of data from the "Survey of solidarity 2010" $(\mathrm{N}=1389)$ generated by the measurement center MIDE UC of the Pontifical Catholic University, we conclude that there are certain variables of social stratification that currently predict and address the solidarity's orientations in Chile. This suggests that the need for solidarity not only responds to a stratified outlining of modern society, but also that different axes of stratification influence the social dimensions of solidarity.
\end{abstract}

Key words: Solidarity, Modernity, Social Stratification, Social Exclusion.

\section{Sobre o estudo empírico da solidariedade: abordagens conceituais e metodológicos}

Resumo: A solidariedade, entendida como uma operação social e comunicativo destinado a preservar a coesão do povo no contexto da sociedade moderna, destaca a persistência de uma ordem social estratificada que produz a exclusão de certos direitos sociais. Este artigo tenta discutir mais a fundo esta definição, oferecendo uma análise empírica e multidimensional das condutas solidarias no Chile. Usando analise bivariada e MANOVA dos dados do "Inquérito solidariedade 2010" ( $\mathrm{N}=1389)$ gerado pela centro da medição do Pontificia Universidade Católica, concluímos que existem certas variáveis __ do esquema de estratificação social que agora permitem prever o rumo e rota de solidariedade no Chile. Isto sugere que a solidariedade não só responde a uma esquematização estratificada dasociedade moderna, mas também acrescenta que os diferentes eixos de estratificação social influenciam as dimensões da solidariedade

Palavras-chave: Solidariedade, Modernidade, a estratificação social, exclusão social.

$$
* * *
$$

\section{Introducción}

La solidaridad es un término con una fuerte carga normativa: "es bueno ser solidario". Generalmente, las aproximaciones teóricas hacia el concepto de solidaridad se encuentran alineadas con esta carga, planteándola como un valor, un principio moral o una cualidad intrínseca de las personas que es necesario potenciar (Dockendorff, 1995; Brugnoli \& Sprovera, 2010; Razeto, 1993, 2001; Pérez Rodríguez, 2007; 
Mardones et al, 1995; Salvat, 2002; Falcón, 1997). Este punto de partida ético y normativo de los estudios de solidaridad ha incidido en que ella sea poco estudiada desde la perspectiva de las ciencias sociales. Los pocos avances que en Chile han existido en este sentido, han sido elaborados por trabajos sociológicos que han propuesto a la solidaridad como una operación social y comunicativa de un sistema de la cooperación destinada a aumentar la probabilidad de inclusión de sujetos excluidos de diversos derechos sociales (Mascareño, 2006; Arnold \& Thumala, 2006). En este trabajo tomaremos esta línea argumentativa para avanzar en la proposición de una noción de solidaridad que sea susceptible de operacionalización y cuantificación en distintas dimensiones, para posteriormente medirla empíricamente a través de los datos de la "Encuesta de Solidaridad 2010" implementada por el centro de medición MIDE UC de la Pontificia Universidad Católica de Chile. Nos enfocaremos finalmente en los factores de estratificación social que inciden en la variación de cada una de estas dimensiones, de tal modo generar una discusión teórica y metodológica del fenómeno de la solidaridad que sintetice aportes de distintas perspectivas sociales como la teoría sistémica y la teoría estructuralista de la sociedad.

El presente artículo comienza proponiendo que la solidaridad corresponde a una operación social y comunicativa moderna destinada a reducir los efectos de la estratificación de la sociedad. Junto a ello se presenta la operacionalización de la solidaridad en diferentes dimensiones, derivada de la definición conceptual esgrimida. En segundo lugar, evidenciamos la relación de ciertos factores de estratificación social que influenciarían las dimensiones de solidaridad. Los datos, variables y métodos utilizados en nuestro trabajo son el tema de la tercera sección, y posteriormente en la cuarta parte se analizan las asociaciones propuestas entre variables de estratificación y dimensiones de solidaridad. Finalmente en las conclusiones se describe el aporte teórico y metodológico de este trabajo para la tematización de la solidaridad como fenómeno social, además de las limitaciones del análisis cuantitativo realizado.

\section{Conceptualización y operacionalización de la solidaridad}

La desigualdad social y la necesidad de la solidaridad como un intento de disminuir las consecuencias desintegradoras de la estratificación de la sociedad, corresponden a procesos que comienzan a describirse solo en la sociedad moderna. Aún cuando en la fase tradicional o pre-moderna existían diferencias sociales entre los distintos segmentos, los individuos eran mantenidos en conjunto por un orden superior religioso, lo que producía que la estructuración de la sociedad no fuera criticada totalmente por cuanto era imposible refutar convencimientos sociales de este tipo (Durkheim, 1985). En el mismo sentido, la noción de solidaridad como una expresión de colaboración hacia los más desfavorecidos por el orden social difícilmente podía ser concebida, pues existía una alta legitimidad religiosa de la segmentación de la sociedad. En dicha fase social las manifestaciones de ayuda eran vivenciadas como parte de la virtud cristiana de la caridad (Nocera, 2007; Stjernø, 2005), la cual no poseía entre sus principios el cuestionamiento de la desigualdad social.

Con el advenimiento de la modernidad, el carácter estratificado de la sociedad comienza a volverse incierto y criticable. La alta posibilidad de diferenciación de sistemas funcionales (política, economía, religión, ciencia, educación, etc.), que emerge con el aumento de complejidad de la sociedad moderna secularizada (Luhmann, 2007; Mascareño, 2010; Valenzuela, 2010), obliga a pensar la noción de desigualdad social como un aspecto problemático de cada espacio funcional de la sociedad, en tanto cada uno de estos ya no se legitiman por principios religiosos, sino que deben proveer oportunidades de integración social para cada individuo (Stichweh, 2004).

En el caso de Chile, rasgos característicos de la modernidad como por ejemplo reforma religiosa, liberalismo democrático, procesos de racionalización social, especialización laboral, heterogeneidad cultural y diferenciación funcional, permiten señalar a este país como una sociedad moderna (PNUD, 1998). Existe un amplio consenso entre los investigadores nacionales respecto de ello, sin embargo existen diferentes posturas para asumir la presencia de la modernidad. Por un lado observamos aproximaciones que desarrollan la distinción entre modernidad e identidad (Morandé, 1984), evidenciando que la modernidad es un proceso esencialmente europeo, por tanto impuesto a la realidad chilena caracterizada por un ethos barroco y mestizo que se sintetiza con esta nueva forma de organización social. Asimismo, se ha señalado que este fenómeno se superpone a una enorme heterogeneidad cultural típica de la expresión autóctona de Chile, generándose una modernidad subordinada (Brunner et. al., 1989) o modernización periférica (Parker, 1995). 
De un modo distinto, se ha reconocido a la modernidad en Chile como un proceso que emerge de la mediación con distintas tradiciones culturales, por lo cual nunca se trata de un fenómeno absolutamente intrínseco y tampoco totalmente foráneo (Larraín, 2001). Finalmente destacamos estudios que, producto del desequilibrio económico y político, identifican el desarrollo de la modernidad en Chile como una modernidad dispareja (Subercaseaux, 1996), una modernidad tardía (Parker, 1995), o una modernidad concéntrica (Mascareño, 2004).

En este nuevo contexto, el concepto de solidaridad es concebido por los primeros cientistas sociales como un vínculo que permite la reducción de la complejidad social que emerge en conjunto con la modernidad. Durkheim (1985) es el primero que define a la solidaridad sociológicamente en esta dirección. A partir de la influencia de los filósofos sociales Pierre Leroux y Auguste Comte, establece que la solidaridad consiste en ciertas pautas de interacción que promueven entendimiento en sociedades industrializadas en las que la alta especialización de los roles de trabajo amenaza frecuentemente la coordinación orgánica de la sociedad. Al definir la solidaridad de este modo, Durkheim no está refiriéndose específicamente al concepto de "donación" o "conductas de ayuda", pues su intención no era profundizar en fenómenos de colaboración sino más bien en los tópicos que sostenían la cohesión y la integración en sociedades modernas. No obstante ello, en virtud de lo planteado por este autor, la solidaridad comienza a ser por primera vez pensada como un concepto posible de tematizar dentro de la tradición de la ciencia social.

En las ciencias sociales se señala que la solidaridad social posibilita el equilibrio en una sociedad moderna con un fuerte componente de individualización que pone en duda constantemente la cohesión entre las personas (De Beer \& Kosteer, 2009; Beck, 2007; Kahane, 1999; Lidz, 1989). La solidaridad también es pensada como un vínculo cívico o ciudadano o como un mediador político entre el individuo y la comunidad, más allá de las obligaciones que la ley esgrime, esto es, tanto en un nivel factual como normativo (Scholz, 2008; Vásquez, 2005; Brunkhorst, 2005; Bayertz, 1999). Es además concebida como un imperativo social que normativiza el comportamiento individual de aquellos sujetos que participan de los intereses colectivos de la sociedad (Komter, 2005; Aluacil, 2004; Hechter, 2001).

Desde la perspectiva sistémica de la sociología, la solidaridad ha sido definida como una operación propia de un sistema de la cooperación. Este sistema posee la función de aumentar la probabilidad de inclusión a individuos excluidos de diferentes derechos sociales (Mascareño, 2006). La noción de excluidos sociales no refiere únicamente a personas pobres, sino que además sugiere la idea de sujetos en condición de miseria social, vale decir, individuos que ocupen posiciones desfavorables en distintos espacios de la sociedad (Bourdieu, 1993). Desde esta aproximación sociológica, la solidaridad no se constituye simplemente desde la acción de un sujeto que posee la expectativa de colaboración, sino además desde la experiencia de la persona destinataria de esa ayuda quien debe utilizarla para lograr inclusión en cualquier sistema social. Este concepto significa entonces una forma condensada de comunicación en la que un individuo aporta libertad de inclusión a otra persona, la cual experimenta tal expectativa del mismo modo y utiliza esa oportunidad para integrarse a un espacio social del cual ha sido excluido/a (Mascareño, 2006). La aproximación sistémica a la solidaridad no solo otorga una nueva perspectiva de análisis sino que nos acerca a la conceptualización operativa del fenómeno de la solidaridad como conductas altruistas o filantrópicas, lo que anteriormente parecía inapropiado desde lo postulado por Durkheim.

La solidaridad precisada desde una aproximación comunicativa permite en primer lugar indicar que ella responde a una demanda de asociatividad y actitudes comunitarias en un contexto social estratificado, que se asumen como relaciones de beneficio, tanto por la persona que posee estas expectativas como por la que las recibe (Arnold \& Thumala, 2006). Sin embargo, si la solidaridad es definida además como una operación social relativa a impedir que la exclusión social altere la interdependencia de los sujetos dentro de una sociedad, esto nos aporta importantes elementos para la operacionalización y posterior medición multidimensional de este concepto. Siguiendo los aportes de Luhmann (2007), sugerimos que cualquier operación social que intente reducir la complejidad de la actual sociedad moderna, es distinguible al menos en tres aspectos o dimensiones distintas: dimensión objetual, dimensión social y dimensión temporal. $\quad$ En el caso de la solidaridad como una operación destinada a la inclusión social, sugerimos la siguiente desagregación dimensional: a) Dimensión objetual o material, vinculada a la donación de objetos o bienes materiales; b) Dimensión social de la solidaridad, relacionada con la donación de dinero de un individuo 
(alter) a otro (ego) privado de ciertas prestaciones sociales a propósito de su baja condición socioeconómica; c) Finalmente una dimensión temporal de la solidaridad, referida a la donación de tiempo en labores o conductas que aporten a la integración social de otros sujetos.

Tradicionalmente, la solidaridad se asocia en términos concretos a una dimensión eminentemente social. Así diferentes estudios empíricos sobre este fenómeno social (como la "Encuesta de Solidaridad 2009" generada por MIDE UC) se han vinculado principalmente a los ámbitos de las donaciones, la participación en colectas y las campañas de ayuda. La "Encuesta de Solidaridad 2010" integra preguntas respecto a la realización de conductas de ayuda agrupadas en las dimensiones donación de dinero, donaciones materiales y donación de tiempo (González, Cortés, Lay, Valencia \& Castillo, 2010). Ello permite vincular la noción teórica multidimensional de la solidaridad con su construcción metodológica.

La operacionalización de la donación de dinero comprende ítems respecto a la frecuencia de donación en diferentes situaciones, como colectas, Teletón y gente en la calle. Paralelamente la dimensión donación material se ha medido a partir de preguntas respecto a la frecuencia se ha colaborado con bienes tales como materiales de construcción, vestimenta y libros. En último término, la donación de tiempo se ha desagregado en diferentes actividades de voluntariado. El detalle de estos ítems se presenta en el apartado 3.2.

\section{Factores de estratificación social asociadas a la solidaridad}

La tesis sobre el condicionamiento estructural del espacio social sobre las disposiciones y conductas de los individuos es un punto central del presente artículo (Kerbo, 2005; Grusky, 1994). Es por ello que nos preguntamos cuáles son y en qué medida se relacionan algunas variables de estratificación social (género, nivel socioeconómico, nivel educacional y edad) con las tres dimensiones propuestas de la solidaridad. Asimismo, nos interesa observar si la constelación de estos factores tiene influencia en la solidaridad dependiendo de la dimensión que se mida. Existen antecedentes sobre la determinación de la edad, el género y el nivel educacional sobre conductas solidarias centradas en la donación de dinero (Castillo, Leal, Madero \& Miranda, 2011). Sin embargo, nos interesa analizar la incidencia de estos y otros factores desde una perspectiva multidimensional de la solidaridad examinando aspectos más allá de las conductas monetarias. Al respecto, algunos autores señalan que existen variables que inciden y moldean las formas de colaboración de los individuos, que comentamos a continuación:

\section{Nivel Socioeconómico}

Stirling (2007) y Pearce (1993) indican que uno de los factores más determinantes de la capacidad de cooperación de las personas dice relación con el nivel socio-económico, esto es, el nivel de ingreso mensual de cada sujeto. Según ellos la acumulación de capital económico y cultural (Bourdieu, 1993), incide directamente en las conductas de ayuda como el voluntariado o la donación.

\section{Género}

El género, entendido como un sistema de características socialmente construidas de hombres y mujeres que determinan las oportunidades y los niveles de acceso a diversos beneficios sociales (Mora, 2009, 2008), es otra de las variables que influencia el grado de participación solidaria. Se ha visto en general que las mujeres son más proclives a ser solidarias que los hombres (Ariño \& Castelló, 2007) y además que donan más dinero y tiempo que ellos (Cox \& Deck, 2006). Otra tendencia interesante en función de este factor de estratificación es que mientras se establece que los hombres poseen un comportamiento menos generoso que las mujeres, éstas poseen cierta afinidad en abocarse a trabajos no-pagados (Gluksmann, 2000), en los que a las personas se les remunera con la valoración de su capacidad de reciprocidad social como por ejemplo en instituciones abocadas a trabajos voluntarios (Migueli, 2007).

\section{Nivel Educacional}

Ilcan \& Bazoc (2004), señalan que el nivel educacional corresponde a una de las variables más determinantes de la solidaridad. Esto por cuanto la mayor cantidad de años de estudio deriva en mayor 
autonomía y madurez individual, por lo tanto mayor capacidad para observar críticamente la realidad social desigual y promover soluciones para ello. A partir de esto los autores señalan que gran parte de las personas que poseen conductas de solidaridad, son personas de un nivel educacional elevado.

\section{Edad}

Finalmente la edad se comporta como una variable determinante de la solidaridad, pues se ha observado que a menor edad mayor es la participación desinteresada (Dávila, 2008; Hager \& Brudney, 2004). Se piensa que las personas adultas al estar inmersos en dinámicas familiares, laborales y profesionales, concentran sus expectativas en esas instancias sociales por lo cual sus conductas solidarias se reducen.

En función de estas investigaciones, proponemos que los factores género, nivel socioeconómico, nivel educacional y edad poseerían influencia sobre las formas que adquiere la solidaridad en Chile, pero que el grado de determinación de cada uno de ellos variará en función de la dimensión de solidaridad que se esté evaluando. Con la aproximación a la definición del concepto de la solidaridad, y a las variables de estratificación que dentro de la literatura se señalan como relevantes, queremos examinar algunas de las prácticas que caracterizan a la solidaridad en nuestro país, o dicho en lo términos conceptuales anteriormente discutidos, indagar en la relación entre las dimensiones creadas de la solidaridad y ciertos factores de estratificación social.

De este modo, los objetivos específicos de este trabajo son cuatro. En primer lugar, describir las medidas de las distintas dimensiones de la solidaridad en Chile, y desarrollar una comparación entre ellas. Al respecto, estimamos a partir de los resultados de Castillo et. al. (2011), que la dimensión donación de dinero será la expresión de solidaridad más presentada. El segundo objetivo específico consiste en analizar en qué medida la donación de dinero se relaciona con otras dimensiones del "Índice de Solidaridad Global 2010" así poder enriquecer el análisis de solidaridad más allá de los donativos monetarios. El tercer objetivo específico comprende la comparación del efecto de los predictores o variables independientes (expuestas en el punto 3.2) en las tres dimensiones de solidaridad y, en cuarto término, observar si ello varía en función de la dimensión de solidaridad seleccionada.

\section{Datos, Variables y Método de Análisis}

\section{Datos}

Para el análisis propuesto utilizaremos los datos de la "Encuesta de Solidaridad 2010", investigación conducida por los investigadores Roberto González y Flavio Cortés del centro de medición MIDE UC de la Facultad de Ciencias Sociales de la Pontificia Universidad Católica de Chile. Esta encuesta fue dirigida a 1389 hombres y mujeres de todos los grupos socioeconómicos, entre 18 y 64 años de edad, residentes en hogares particulares del Gran Santiago y las principales ciudades de Chile (Antofagasta, Viña del Mar, Valparaíso, Concepción, Talcahuano y Temuco). La población representada fue 5,1 millones de personas según las proyecciones de población para el año 2010 del INE-CELADE (basado en el Censo de 2002). El método de recolección de información fue a través de entrevistas cara a cara en hogares particulares aplicando una tabla aleatoria tipo Kish, la cual guío la clasificación de la muestra en sus tres etapas (manzana-hogarpersona) con muestreo aleatorio y estratificado por GSE en cada ciudad. El error muestral se estima en $\pm 2,6 \%$ considerando varianza máxima y un $95 \%$ de confianza. La recolección de datos se efectuó entre el 4 de Septiembre y el 6 de Octubre de 2010.

\section{Variables}

Las variables de este estudio consisten en 3 variables dependientes (tabla 1), que corresponden a cada una de las dimensiones de la "Encuesta de Solidaridad 2010", y 4 variables independientes (tabla 2), derivados de los 4 factores de estratificación seleccionados en el presente artículo.

Tabla 1: Variables Dependientes 


\begin{tabular}{|c|c|}
\hline Variable & Operacionalización \\
\hline 1.-Dimensión Donación Dinero ( $\alpha$ cronbach 0,78$)$ & $\begin{array}{l}\text { Cada ítem de este índice fue medido con la siguiente escala: } \\
\text { 1: Nunca o casi nunca, 2: Rara vez, 3: Algunas veces, 4: Algunas veces, } \\
\text { 5: Siempre o casi siempre. } \\
\text { 1a Donar parte de su vuelto. } \\
\text { 1b Dar dinero en catástrofes } \\
\text { 1c Dar dinero a instituciones } \\
\text { 1d Dar dinero a la teletón } \\
\text { 1e Dar dinero en colectas nacionales } \\
\text { 1f Dar dinero a personas en la calle }\end{array}$ \\
\hline $\begin{array}{l}\text { 2.-Dimensión Donación Materiales ( } \alpha \text { cronbach } \\
0,81 \text { ) }\end{array}$ & $\begin{array}{l}\text { Cada ítem de este índice fue medido con la siguiente escala: } \\
\text { 1: Nunca o casi nunca, 2: Rara vez, 3: Algunas veces, 4: Algunas veces, } \\
\text { 5: Siempre o casi siempre. } \\
\text { 2a Mercadería y alimentos } \\
\text { 2b Pañales y ropa para niños } \\
\text { 2c Artículos de limpieza, aseo personal } \\
\text { 2d Materiales de construcción } \\
\text { 2e Ropa, zapatos, frazadas } \\
\text { 2f Libros, juguetes, útiles escolares } \\
\text { 2g Medicamentos } \\
\text { 2h Muebles }\end{array}$ \\
\hline 3.-Dimensión Donación Tiempo ( $\alpha$ cronbach 0,75$)$ & $\begin{array}{l}\text { Cada ítem de este índice fue medido con la siguiente escala: } \\
\text { 1: Nunca o casi nunca, 2: Rara vez, 3: Algunas veces, 4: Algunas veces, } \\
\text { 5: Siempre o casi siempre. } \\
\text { 3a Dar apoyo emocional en hogares de acogida, } \\
\text { 3b Visitar personas en hogares } \\
\text { 3c Trabajar gratuitamente en organizaciones de voluntariado } \\
\text { 3d Dar alojamiento en el hogar a personas pobres } \\
\text { 3e Participar en actividades benéficas } \\
\text { 3f Preparar/Servir comida a personas } \\
\text { 3g Dar clases gratuitas } \\
\text { 3h Construir/Reparar viviendas }\end{array}$ \\
\hline
\end{tabular}

Fuente: Encuesta de Solidaridad, 2010, www.mideuc.cl

\section{Tabla 2: Variables Independientes}

Variable $\quad$ Operacionalización

1.Nivel Socio Económico 1 1a. Valores promedio de variable categórica "tramos de ingreso mensuales hogar" se divide por variable "cantidad de personas que viven permanentemente en hogar" para obtener el ingreso neto equivalente de cada sujeto. Esto se recodifica en 5 tramos de ingreso correspondientes a los quintiles de ingreso.

2.Edad

3.Nivel Educacional del encuestado

4.Género 2a. Variable recodificada en 4 categorías de edad siendo 1: ESPECIFICAR AÑOS joven, 2:adulto-joven, 3: adulto mayor, 4: adulto mayor.

1: Educación Básica Completa,

2: Educación Media Incompleta,

3: Educación Media Completa,

4: Educación Técnica Completa,

5: Educación Universitaria Incompleta,

6: Educación Universitaria Completa.

4a. Variable dicotómica sexo con valores 1: Hombre y 0: Mujer.

Fuente: Encuesta de Solidaridad en Chile, 2010, www.mideuc.cl 


\section{Método de análisis}

En primer lugar se abordan las medidas respectivas de las tres dimensiones de solidaridad propuestas. Luego evidenciamos las relaciones bivariadas estadísticamente significativas entre nuestras tres variables dependientes y las variables de estratificación seleccionadas. Finalmente se genera un análisis MANOVA tomando en cuenta el efecto conjunto de las varianzas de las tres variables dependientes. Estas exploraciones se desarrollan mediante el software de análisis cuantitativo Stata/SE v.11.

\section{Análisis}

De acuerdo a los análisis de González et. al., (2010), las medidas de las tres dimensiones de la solidaridad varían entre 0 y 10 (gráfico 1), así como también el "Índice de solidaridad global", conformado por todos los ítemes de las tres dimensiones. El valor 0 representa la ausencia total de conductas de ayuda y 10 representa el máximo nivel de ayuda posible. En el total de la muestra, se constató que el "Índice de Solidaridad Global 2010" obtuvo un puntaje promedio igual a 3.3. Este puntaje se encuentra bajo el punto medio de la distribución total (5.0). Sin embargo, se observó un alto puntaje en la dimensión de donación de dinero (6.0), mientras que las dimensiones de donación de materiales y de tiempo personal reportaron 2.2 y 2.0 puntos respectivamente (González, et al. 2010). La preponderancia de conductas de ayuda ligadas a la donación de dinero ha sido caracterizada como "economía de solidaridad" (Razeto, 2001, 1993) o "solidaridad de mercado" (Brugnoli \& Spravora, 2010), donde aspectos de donación monetaria ocupa un lugar central en las formas de cooperación.

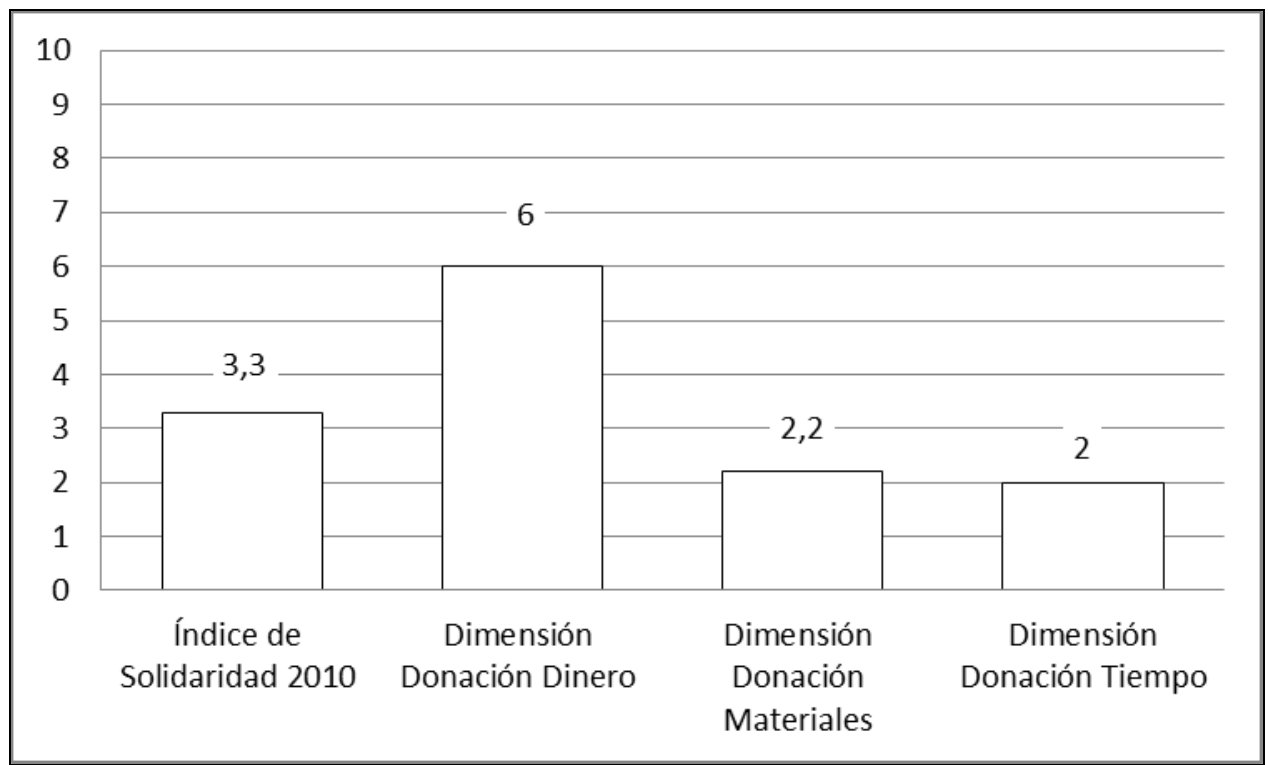

Gráfico 1: Promedio de Indice Solidaridad Global y Dimensiones, adaptado de González et al. (2010)

En cuanto a las correlaciones entre las dimensiones de la solidaridad reportadas en la tabla 3 , observamos que dan cuenta de una asociación moderada, siendo la donación de tiempo la que presenta una menor asociación con las otras dos dimensiones. Este nivel de asociación nos sugiere que las conductas solidarias no necesariamente se manifiestan de modo conjunto en los sujetos, pudiendo existir individuos con un alto compromiso en términos de tiempo y donación materiales, pero sin estar acompañado de una conducta de donación de dinero (y viceversa).

Tabla 3: Correlación Dimensiones Índice de Solidaridad 


\begin{tabular}{lccc}
\hline & $\begin{array}{c}\text { Donación } \\
\text { Dinero }\end{array}$ & $\begin{array}{l}\text { Donación } \\
\text { Materiales }\end{array}$ & $\begin{array}{l}\text { Donación } \\
\text { Tiempo }\end{array}$ \\
\hline Donación Dinero & 1.00 & - & - \\
Donación Materiales & $0.39^{* *}$ & 1.00 & - \\
Donación Tiempo & $0.27^{* *}$ & $0.37^{* *}$ & 1.00 \\
\hline
\end{tabular}

Fuente: Elaboración propia. $* *=\mathrm{p}<0.01$

En cuanto a asociaciones de carácter bivariado con las variables independientes del estudio, observamos que las mujeres poseen en general una mayor disposición a ayudar que los hombres. En el caso de la donación de dinero las mujeres poseen un promedio de 6.5 frente a un 5.9 de los hombres $(\mathrm{t}=4.31$, $\mathrm{p}<$ $0.01)$, diferencia similar a la observada en la donación de materiales $(t=5.24, p<0.01)$, mientras que en donación de tiempo los hombres muestran un promedio de 1.9 frente a un 2.1 de las mujeres $(\mathrm{t}=-2.00$, $\mathrm{p}<0.05)$. Este resultado se vincula a la definición del rol femenino con una mayor disposición y preocupación de individuos excluidos socialmente (Langford \& Mckinnon, 2000; Twenge, 1997). De este modo la división de géneros creada cultural y arbitrariamente poseería sus efectos también en este espacio del mundo social justificando esta diferencia de conductas de ayuda entre mujeres y hombres en la distinción natural entre sexos (Coleman \& Hong, 2008; Yaremko \& Lawson, 2007).

En términos del nivel educacional, observamos que mayores niveles de esta variable se asocian con mayor proclividad a conductas solidarias. Se aprecia que los universitarios con su carrera finalizada o en vías de hacerlo, poseen al menos 0.7 puntos más que las personas con educación básica en el "índice de solidaridad global 2010" ( $\mathrm{p}<0.01)$. En el caso de la donación de dinero esta misma diferencia se amplía a 2 puntos, mientras la donación de tiempo es la dimensión que menos diferencias muestra según nivel educacional, siendo solamente significativa entre aquellos con educación básica y personas con estudios universitarios $(\mathrm{t}=3.18, \mathrm{p}<0.01)$.

La parte final del estudio utiliza la técnica multivariada MANOVA dada su capacidad para observar si existen influencias significativas entre nuestros factores de estratificación y las tres dimensiones de solidaridad simultáneamente. Los análisis a presentar reportan efectos en base al indicador lambda de Wilks. Los resultados de la estimación (tabla 4) revelan en primer lugar que el modelo general es estadísticamente significativo, es decir, existe una asociación general entre los predictores y las tres variables dependientes, $F_{(75,3274)}=2.93, \mathrm{p}<0.00$. Atendiendo a los efectos de cada variable independiente, la evidencia apoya lo observado en términos descriptivos en cuanto al efecto de la variable género, $F_{(3,1095)}=12.31, \mathrm{p}<0.00$, indicando que las mujeres se diferencian de los hombres en las tres dimensiones de la solidaridad en forma conjunta. Los tramos de edad también nos señalan diferencias estadísticas, $F_{(9.2665)}=3.19, \mathrm{p}<0.00$, mientras el efecto del nivel de escolaridad constituye la tercera de las variables consideradas que posee una relación con las conductas de ayuda $F_{(15,3011)}=2.22, \mathrm{p}<0.00$.

Dadas las hipótesis relacionadas con estatus y solidaridad, llama la atención que una variable tan relevante al respecto como es el nivel socioeconómico carezca de un efecto significativo. Análisis adicionales de MANOVA considerando sólo el nivel socioeconómico señalan que existen diferencias significativas entre quintiles de ingreso en relación a conductas solidarias $F_{(12,2992)}=5.25, \mathrm{p}<0.00$, efecto que desaparece al considerar simultáneamente el nivel educacional. Tal situación es consistente con análisis realizados con la versión previa de la encuesta de solidaridad (Castillo, et. al., 2012), donde la influencia del ingreso sobre las donaciones de dinero se anula al momento de ingresar educación en modelos de regresión simple, lo cual se vincula a la estrecha asociación existente entre nivel educacional e ingreso en el país. Finalmente, el análisis considera efectos de interacción entre género y variables de estatus, ya que se podría suponer las grandes brechas de estatus socioeconómico por género en Chile podrían estar asociadas a la influencia del género en las conductas solidarias, pero según nuestros análisis esta interacción en ambos casos no es significativa.

\section{Tabla 4: MANOVA. Variables dependientes en covarianza con variables de estratificación}




\begin{tabular}{lcccc}
\hline Variable & $\mathrm{G}^{\circ} \mathrm{L}$ & $\mathrm{F}(\mathrm{df} 1, \mathrm{df} 2)$ & $\mathrm{F}$ & Prob $>\mathrm{F}$ \\
\hline Modelo & 22 & $66.0,3279.8$ & 3.10 & 0.00 \\
\hline Nivel Socio Económico & 4 & $12.0,2905.3$ & 1.51 & 0.11 \\
\hline Edad & 3 & $9.0,2672.4$ & 3.22 & 0.00 \\
\hline Género & 1 & $3.0,1098.0$ & 13.38 & 0.00 \\
\hline Nivel Educacional & 5 & $15.0,3031.5$ & 2.22 & 0.00 \\
\hline $\begin{array}{l}\text { Nivel Socio Económico * } \\
\text { Género }\end{array}$ & 4 & $12.0,2905.3$ & 1.72 & 0.06 \\
\hline Nivel Educacional * Género & 5 & $15.0,3031.5$ & 1.16 & 0.29 \\
\hline
\end{tabular}

Fuente: Elaboración propia.

\section{Conclusiones}

El concepto de solidaridad definido en primer término como una operación eminentemente moderna que permite a los sujetos estar vinculados socialmente y, en segundo lugar, como una operación social del sistema de la cooperación destinada a integrar a los individuos excluidos de diferentes derechos sociales, constituye un aporte desde la sociología por cuanto nos permite observar las consecuencias sociales de la permanencia de la estratificación en el marco de la sociedad moderna. Este mismo enfoque aportó elementos claves para la operacionalización de la solidaridad en tres dimensiones (donación monetaria, donación de materiales y donación de tiempo). La revisión y el posterior análisis de los datos de la "Encuesta de Solidaridad 2010" proporcionan un sustento al planteamiento sobre la desagregación dimensional de la solidaridad, la jerarquización de las mismas dimensiones y la confiabilidad estadística de la agrupación de indicadores en cada una de ellas.

Adicionalmente exploramos diversos factores de estratificación social que eventualmente podrían condicionar las conductas solidarias de los chilenos. Al aplicar un análisis bivariado, observamos los factores más predictivos de las formas de solidaridad fueran género y nivel educacional. Sin embargo, esto varió al estimar el modelo multivariado MANOVA, donde se aprecia que las variables finalmente significativas son género, edad y nivel educacional. Evidenciar que existen variables de estratificación social que en la actualidad direccionan y predicen la orientación de la solidaridad en Chile, constituye el avance central de este trabajo. Esto pues se establece no solamente que la solidaridad responde a una esquematización estratificada de la sociedad moderna, sino además agrega que diferentes ejes de estratificación social determinan las dimensiones o expresiones de la solidaridad.

La contribución teórica y metodológica de este trabajo resulta del análisis del fenómeno de la cooperación y la solidaridad, desde una perspectiva sociológica asociado a los aportes de la tesis de estratificación. Al respecto, entender este concepto como una operación social y comunicativa destinada a mantener la cohesión de las personas en distintos campos sociales, tanto en una dimensión material, como social y temporal, determinada a su vez por la existencia de un marco de estratificación de la sociedad, promueve una forma alternativa de comprensión de este hecho social, en base a lo señalado por otros investigadores (Mascareño, 2006: Arnold \& Thumala, 2006). Esto pues comprendemos que el objeto de estudio de una investigación sobre solidaridad, refiere ineludiblemente a la definición de ella como una operación social determinada por variables de estratificación, lo que permite medirla empíricamente y revisar los determinantes de las diferencias entre sus dimensiones.

Solo desde la complementación de estas perspectivas fue posible observar que la composición de las dimensiones de la solidaridad son distinguibles en virtud de la influencia de los factores género, nivel educacional y edad. A partir de esto, resulta apresurado hablar de una única forma de la solidaridad, 
proponemos en cambio determinar que existen distintas formas de solidaridad que poseen una estructura que es describible y medible si atendemos a la matriz de estratificación social sobre la cual se esgrime el mismo fenómeno.

Finalmente, queremos comentar ciertas limitantes de este estudio. Si bien hemos comprobado la efectiva influencia de ciertas variables sociodemográficas en 3 dimensiones de la solidaridad en Chile, no obstante también hemos visto que el nivel predictivo de esa relación es relativamente bajo. En consecuencia, pensamos que el análisis de los determinantes de prácticas solidarias se podría enriquecer aún más si se incluyeran variables psicosociales tales como creencias sobre la justicia y la distribución del ingreso, atribuciones de la desigualdad económica, expectativas de patriotismo, entre otras, de tal modo examinar otro tipo de factores explicativos. Además creemos que este análisis se enriquecería complementando el análisis cuantitativo realizado en el presente trabajo, con aproximaciones metodológicas cualitativas que sean capaces de rescatar representaciones y significaciones que actualmente poseen chilenos/as relativas a la temática de la solidaridad, sus dimensiones y sus determinantes sociales, y así perfeccionar el análisis de este fenómeno social.

\section{Nota}

* Este trabajo se enmarca en la investigación "Radiografía de la Solidaridad en Chile", generada y financiada por el centro de investigación MIDE UC de la Pontificia Universidad Católica de Chile.

\section{Bibliografía}

Arnold, Marcelo \& Thumala, Daniela (2006), "Colaboración, participación y sociedad civil en Chile: reflexión para la acción”, En Persona y Sociedad, N²0(1), p.143-156.

Bayertz, Kurt (1999), Solidarity, Kluwer Academic Publishers, Boston, 350p.

Beck, Ulrich (2007), "Beyond class and nation: reframing social inequalities in a globalizing world". En The British Journal of Sociology, N58(4), p.679-705.

Bourdieu, Pierre (1993), La miseria del mundo, Akal, Barcelona, 564p.

Brugnoli, José \& Sprovera, María (2010), "La solidaridad de mercado y sus sujetos en el capitalismo de consumo: un análisis del discurso neoliberal sobre piezas de publicidad en Chile", En Psicologia \& Sociedade, $\mathrm{N}^{\circ} 22(2)$, 247-258.

Brunkhorst, Hauke (2005), Solidarity: from civic friendship to a global legal community, MIT Press, Cambridge, 262p.

Brunner, José Joaquín; Barrios, Alicia \& Catalán, Carlos (1989), Chile, transformaciones culturales y modernidad, FLACSO, Chile, 228p.

Castillo, Juan Carlos; Leal, Paola; Madero, Ignacio \& Miranda, Daniel (2011), “Son los chilenos igualmente solidarios? Análisis de diferencias individuales en las donaciones de dinero", En Prensa, Revista Opinao Publica.

Coleman, Jill \& Hong, Ying-yi (2009), "Beyond Nature and Nurture: The Influence of Lay Gender Theories on Self-stereotyping”, En Self and Identity, N7(1), p.34 - 53.

Cox, James \& Deck, Cary (2006), When are women more generous than men?, Andrew Young School of Policy Studies Research Paper Series No. 06-39 Social Science Electronic Publishing.

Dávila, María (2008), “Abandono del voluntariado, Tasas de abandono y causas más frecuentes”. Comunicación e Ciudadanía, N5.

De Beer, Paul \& Kosteer, Ferry (2009), Sticking Together or falling apart? Solidarity in an Era of Indiviualization and Globalization, University Press, Amsterdam, 246p. 
Dockendorff, Cecilia (1995), Solidaridad: La construcción social de un anhelo, UNICEF/MIDEPLAN/FOSIS, Santiago de Chile, 190p.

Durkheim, Emile (1985), La división del trabajo social, Planea-Agostini, Barcelona, 492p.

Falcón, Enrique (1997), Dimensiones políticas del voluntariado. De la promoción al cambio de estructuras, Cristianisme i Justicia, Barcelona, 21p.

González, Roberto; Cortés, Flavio; Lay, Siugmin; Valencia, Edgar; Castillo, Juan Carlos (2010), ponencia exhibida en presentación "La solidaridad en tiempos normales y tiempos de catástrofe", disponible en: http://www.mideuc.cl/docs/solidaridad2.pdf

Hager, Mark \& Brudney, Jeffrey (2010), Volunteer Management Practices and Retention of Volunteer, The urban Institute, Whashington, 16p.

Hechter, Michael (2001), "Sociology of Solidarity", International Encyclopedia of the Social and Behavioral Sciences, edited by N. J. Smelser and P. B. Baltes, Elsevier, New York, 898p.

Ilcan, Susan \& Basok, Tanya (2005), "Community Government: Voluntary Agencies, Social Justice, and the Responsibilization of Citizens”, En Citizenship Studies, N²8), p.129-144.

Kahaen, David (1999), "Diversity, solidarity and civic friendship", En The Journal of Political Philosophie, $\mathrm{N}^{\circ} 7(3)$, p.267-286.

Komter, Aafke (2005), Social solidarity and the gift, Cambridge University Press, New York, 246p.

Langford, t. \& Mackinnon, J. "The Affective Bases for the Gendering of Traits: Comparing the United States and Canada”, En Social Psychology Quarterly, 2000, N63(1), p.34-48.

Lidz, Victor (1989), "Influence et solidarité, définir un fondement théorique à la sociologie", En Sociologie et sociétés, $\mathrm{N}^{\circ} 21(1), \mathrm{p} .117-142$.

Luhmann, Niklas (2007), La sociedad de la sociedad, Ediciones Herder, México, 954p.

Mascareño, Aldo (2004), “Sociología del derecho (chileno y latinoamericano)», En Persona y sociedad, №18(2), pp. 63-95.

Íderm (2006), "Sociología de la solidaridad, La diferenciación de un sistema de la cooperación”, En Colaboración, cultura y desarrollo, Arnold, Marcelo y Daniela Thumala, Ediciones Universidad de Chile, Santiago de Chile, 246p.

Íderm (2010), Diferenciación y Contingencia en América Latina, Ed. Universidad Alberto Hurtado, Santiago de Chile, 329p.

Mardones, José, Briones, Luis, Fuentes, Pedro \& Cortina, Adela (1997), Hacia una Cultura de la Solidaridad, Caritas, Madrid, 254p.

Mora, Claudia (2008), “Globalización, Género y Migraciones”, En Polis, Revista de la Universidad Bolivariana, $\mathrm{N}^{\mathrm{o}} 7$.

Íderm (2009), "Estratificación social y migración intrarregional: algunas caracterizaciones de la experiencia migratoria en Latinoamérica”, En Universum, N²4(1), p.128-143.

Morandé, Pedro (1984), Cultura y modernidad en América Latina, Cuadernos del instituto de Sociología Pontificia Universidad Católica de Chile, Santiago de Chile, 181p.

Nocera, Pablo (2007), "Aproximaciones para una historia de la formulación sociológica del concepto de solidaridad. Su trayectoria conceptual en el pensamiento francés hasta la sociología de Émile Durkheim”, ponencia presentada en IV jornada de jóvenes investigadores Universidad de Buenos Aires, 19p.

Parker, Cristián (1995), "Identidad, modernización y desarrollo local”, En Revista Academia N¹(1), pp. 43-56. 
Pearce, Jone (1993), Volunteers: The Organizational Behavior of Unpaid Workers, Routledge, Londres, 223p.

PNUD (1998), Informe de Desarrollo Humano 1998: Las paradojas de la modernización, PNUD, Chile

Razeto, Luis (1993), Los caminos de la economía de la solidaridad, Paidós, Barcelona, 131p.

Íderm (2001), "Desarrollo económico y economía de la solidaridad. El desarrollo como expansión, transformación y perfeccionamiento de la economía del tiempo", En Polis, Revista de la Universidad Bolivariana, $\mathrm{N}^{\circ} 1$.

Salvat, Pablo (2002), El porvenir de la equidad. Aportaciones para un giro ético en la filosofía política contemporánea, LOM/Universidad Alberto Hurtado, Santiago de Chile, 232p.

Scholz, Sally (2008), Political solidarity, Pennsylvania State University Press, Pennsylvania, 286p.

Stjernø, Steinar (2005), Solidarity in Europe: the history of an idea, Cambridge University Press, New York, 392 p.

Stichweh, Rudolf (2004), On the Genesis of World Society: Innovations and Mechanism, Universität Luzern, Lucerna, 24p.

Stirling, Christine (2007), The Volunteer Citizen, Health Services and Agency: The Identity Work of Australian and New Zealand Ambulance Volunteers, Thesis of Doctor of Philosophy, University of Tasmania, 329p.

Subercaseaux, Bernardo (1996), Chile, ¿un país moderno?, Ed. Zeta, Barcelona, 205p.

Twenge, Jean (1997), "Changes in Masculine and Feminine TV-aits Over Time: A Meta-Analysis", En Sex Roles, $\mathrm{N}^{\circ} 36(5), \mathrm{p} 305-325$.

Valenzuela, Fernando (2010), The guild of painters in the evolution of art in colonial Cusco, Workingpaper des Soziologischen Seminars, Soziologisches Seminar der Universität Luzern, Lucerna, 40p.

Vásquez, Adolfo (2005), "Rorty: pragmatismo, ironismo liberal y solidaridad", En Polis, Revista de la Universidad Bolivariana, $\mathrm{N}^{\circ} 11$.

Yaremko, Sara. \& Lawson, Karen (2007), “Gender, Internalization of Expressive Traits, and Expectations of Parenting”, En Sex Roles, N57, p.675-687.

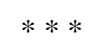

Recibido: 02.08.2011

Aceptado: 23.11.2011 